\title{
Zwischen Heimatverlust und unsicherer Gegenwart: Sozialer Wandel in Zonenkinder (2002) von Jana Hensel und Scotch (2010) von Ioana Bradea
}

\section{Roxana-Andreea GHIŢĂ}

Lekt. -Dr.; Universität Craiova; E-Mail: roxaneghita@gmail.com

\begin{abstract}
My paper will explore the interrelation between past, present and identity, as well as the dynamics of social change in contemporary German and Romanian literature, as exemplified by Jana Hensel's Zonenkinder (2002) and Ioana Bradea's Scotch (2010). Both authors belong to a new generation of writers who, having experienced the collapse of the communist regime as adolescents, investigate the traumatic experience of change and adjustment to the social, economic and cultural realities of post-communist societies. While Hensel aims at recreating the lost Heimat (motherland) as an Erinnerungsraum (space of remembrance) and portraying the social tensions of the post-unification decade from an Eastern German perspective, Bradea focuses on depicting the desolate post-communist industrial landscape, as well as the everyday lives of anonymous Romanians caught in the vagaries of transition.
\end{abstract}

Keywords: Jana Hensel, Eastern Germany, memory, social change, IoanaBradea, Romania, post-communist industrial landscape

Seit dem Zusammenbruch des Ostblocks durchlaufen die postsozialistischen Länder gesellschaftliche, politische und kulturelle Transformationsprozesse, die von ähnlichen Entwicklungstendenzen geprägt sind. Der postsozialistische Wandel hat 
„unerwartet einen krisenhaften Verlauf" genommen, der die ursprüngliche Begeisterung für die Marktwirtschaft und westliche Werte in Enttäuschung, Verwirrung und Angst umschlug. Piotr Sztompka, ein führender Soziologe auf dem Gebiet des sozialen Wandels, notierte zur traumatischen Dynamik der Umwälzungen: „Changing from Homo Sovieticus to the modern Westerners, abandoning the crippled and deficient East European identity and acquiring a full-fledged, proud European identity required time". ${ }^{2}$

Eine neue Generation von Autoren, die ihre Kindheit und Jugend im kommunistischen Regime verbracht haben, reagierte überall im ehemaligen Ostblock auf den gesellschaftlichen Umbruch, indem sie in ihren Werken den Verlust der vertrauten Lebensverhältnisse, die individuellen und kollektiven Veränderungsprozesse und die Unsicherheit der Gegenwart verarbeiteten. Meine Untersuchung widmet sich der Frage des sozialen Wandels in den Prosawerken Zonenkinder (2002) von Jana Hensel und Scotch (Klebeband, 2010) von Ioana Bradea, wobei die unterschiedlichen Herangehensweisen der deutschen und rumänischen Schriftstellerin an den Themen wie Heimatverlust, Identität und Transformation im Mittelpunkt stehen.

\section{Zonenkinder von Jana Hensel: die schmerzvolle Trennung von der verschwundenen Heimat}

Die enge Verknüpfung zwischen Identität und (Erinnerungs-) Raum kommt vielleicht nirgendwo besser zum Vorschein als in

${ }^{1}$ Müller, Klaus: Einleitung. In: Müller, Klaus (Hg.): Postsozialistische Krisen: Theoretische Ansätze und empirische Befunde. Wiesbaden 2013, S. 7-9, hier S. 8.

2 Sztompka, Piotr: The Ambivalence of Social Change in Post-Communist Societies. The Case of Poland. In: Soeffner, Hans-Georg (Hg.): Unsichere Zeiten. Herausforderungen gesellschaftlicher Transformationen. Wiesbaden 2010, S. 265-286, hier S. 266. 
Jana Hensels Erinnerungsbuch Zonenkinder ${ }^{3}$. Ein untergegangenes Land, die DDR, soll von der 1976 geborenen und in Leipzig aufgewachsenen Autorin im Medium der Literatur als Erinnerungsraum für die Zukunft (re)konstruiert und aufbewahrt werden. Zwar handelt es sich um eine subjektive, keinen Totalitätsanspruch erhebende Perspektive, doch inszeniert sich Hensel nichtsdestoweniger als Stellvertreterin einer ganzen Generation: Die kollektive Ausrichtung wird in erster Linie durch das Wechselspiel der Erzählinstanzen, vom Ich zum verallgemeinernden Wir, konstruiert. ${ }^{4}$

Bereits im Titel wird das Selbstverständnis einer Generation an eine räumliche Bezeichnung gebunden: die Zone. Im transdisziplinären Sammelband Die Generation der Wendekinder: Elaboration eines Forschungsfeldes bezeichnen Bahr/Lorek in ihrem Versuch, die „3. Generation Ostdeutscher" zu definieren, Hensels Buch als prägend für die Entstehung und Verbreitung des „Wendekinder“-Begriffs:

Mit der Veröffentlichung von Jana Hensels Zonenkinder trat 2002 erstmalig der Begriff der, Wendekinder' in die Öffentlichkeit, welcher all diejenigen beschrieb, welche die Jahre 1989 und 1990 als Kinder und Jugendliche erlebt hatten. Die Erscheinung von Hensels Autobiografie hatte zur Folge, dass in den folgenden Jahren weitere, ähnliche Werke und semi-autobiografische Lebenserzählungen von jüngeren Ostdeutschen entstanden. ${ }^{5}$

${ }^{3}$ Die folgenden Ausführungen zu Zonenkinder basieren auf einem Kapitel meines Buches Literatur und Geschichte. Fallstudien zur narrativen Aufarbeitung der Vergangenheit im deutschen und europäischen Kontext. Craiova 2018.

${ }^{4}$ Dieser Bestseller hat eine leidenschaftliche Debatte um gedächtniskulturelle und -politische Fragestellungen entfacht.

5 Bahr, Katrin/Lorek, Melanie: „Ja, wohingehen sie denn?“: die „3. Generation Ostdeutscher" zwischen Suchen und Finden am Beispiel des 1.5 Generationskonzeptes. In: Lettrari, Adriana/Christina Nestler/ Nadja Troi-Boeck (Hgg.): Die Generation der Wendekinder: Elaboration eines Forschungsfeldes. Wiesbaden 2015, S. 255-278, hier S. 
Durch den Begriff Zone, ein für die sowjetische Besatzungszone und nach 1949 in der BRD auch für die DDR verwendetes Kürzel, wird auf ein Staatsgebilde verwiesen, für das die geopolitische Ost-West-Dichotomie als Gründungs- und Identitätsbegründung gilt. Hensels Text zielt auf die Rekonstruktion der DDR als Herkunft- und Lebensraum für sie und ihre Generation, d.h. als erlebtes Zeit-Raum-Gefüge, ab:

„Es ging mir darum, Erinnerung ideologisch zu entschlacken. Ich wollte von der DDR nicht wie von einem politischen System erzählen. Ich wollte sie als einen Herkunftsraum beschreiben" ${ }^{\text {“6 }}$.

Was die Zonenkinder charakterisiert, ist die schmerzhafte, paradoxe Erfahrung, sich nach 1989 mit einer doppelten Art von Fremdheit auseinandersetzen zu müssen. Durch den rasanten gesellschaftlichen Wandel befanden sie sich einerseits quasi über Nacht im fremden Westen. ${ }^{7}$ Die Überlegenheit des Westens gegenüber dem Osten wurde nach der Wende zum Axiom der neuen kulturellen Ordnung ${ }^{8}$, der Hensels Generation sich anzupassen versuchte, wie auch zur überwältigenden Alltagsrealität, was zu einschneidenden, traumatischen Ereignissen führte:

258. Im Vergleich zum Begriff,,Wendekinder" sei, so Bahr/Lorek, die Bezeichnung „3. Generation Ostdeutscher“ viel präziser. Sie beziehe sich auf all diejenigen, ,die zwischen 1975 und 1985 geboren wurden [...] und die demnach die deutsche Wiedervereinigung in einem Alter zwischen fünf und 15 Jahren erlebten“" (ebd.).

${ }^{6}$ Hensel in: Kraushaar, Tom: Die Zonenkinder und Wir-Die Geschichte eines Phänomens. Reinbek bei Hamburg 2004, S. 94.

7 Vgl. auch Hensel, Jana: Zonenkinder. Reinbek bei Hamburg 2002, S. 45: „Wie ich waren auch sie [Hensels ostdeutsche Freunde, Anm. d. Verf.] bemüht, sich dauerhaft in einer Fremdheit einzurichten, die sich auf dem Boden des Heimatlandesausbreitete und von uns verlangte, permanent alte gegen neue Bilder auszutauschen“.

8 Ebd., S. 73: „Wir waren die Söhne und Töchter der Verlierer, von den Gewinnern als Proletarier bespöttelt, mit dem Geruch von Totalitarismus und Arbeitsscheu behaftet". 
Wir schauten uns die neue Bundesrepublik genau an, dachten häufig über uns nach und grübelten nach Wegen, wie wir am wenigsten auffallen und trotzdem am weitesten kommen könnten. Ganz unverhofft sozusagen wollten wir eines Tages auftauchen, unseren Pass hochhalten, in denen man Geburtsorte wie Cottbus, Sonneberg oder Wismar würde lesen können, und alle anderen in Staunen versetzen, dass wir es mit einem so gravierenden Schönheitsfehler gleichwohl geschafft hatten. ${ }^{9}$

Bezeichnenderweise lautet der Titel des letzten Kapitels Go West. Über unsere Zukunft, wo eine Art Bilanz des Prozesses „Abschied von unserem Heimatland zu nehmen“10 gezogen wird.

Andererseits ging mit dem Verlust der Heimat auch das Verlorengehen der eigenen Vergangenheit einher, sodass das eigene Leben bis zur epochalen Zäsur des Mauerfalls ebenfalls zum fremden, fast unzugänglichen Zeit-Raum-Gebilde wurde. Stellt die Kindheit aus der Perspektive des Erwachsenen-Ich generell eine abgeschlossene, unwiederbringliche Lebensphase dar, so gewinnt die Verlusterfahrung für Hensels Generation dadurch spezielle Schärfe, dass mit dem Ende der Kindheit auch die Lebensräume, an die diese Kindheit gekoppelt ist, zu existieren aufgehört haben. Valeska Steinig zufolge vermag Hensel nicht zu erkennen, dass die Wiederbringung vergangener Zeit ,aus rein biografischen Gründen nicht möglich ist". ${ }^{11}$ Dabei unterschätzt sie meines Erachtens die Partikularität der Umbruchserfahrung dieser Generation, der die Heimat nicht nur zeitlich, sondern auch räumlich und kulturell abhanden gekommen ist. Diese belastende Erfahrung wäre daher eher als kulturelles Psychotrauma anzusehen, wie Martina Caspari in Anlehnung an die Kulturschocktheorie wie auch unter

9 Ebd., S. 98 f.

${ }^{10}$ Ebd., S. 167.

${ }^{11}$ Steinig, Valeska: Abschied von der DDR - Autobiografisches Schreiben nach dem Ende der politischen Alternative. Frankfurt am Main 2007, S. 141. 
Berücksichtigung der einmaligen geopolitischen Dynamik der Wende vorschlägt. ${ }^{12}$ Parallel zu realen Raum-Zeit-Bezügen tritt demzufolge ein Bild der Heimat als emotionaler Zustand (Heimatgefühl), als imaginierter und idealisierter „Kompensationsraum"13 für den Verlust der Heimat in der wirklichen Welt in Erscheinung. Diese ,Eliminierung' der Heimat wird von Hensel auch als Auslöschung der eigenen Kindheit, die durch den Einbruch unverständlicher Mächte in ihre bis dahin als idyllisch wahrgenommene Lebenswelt bewirkt wurde, empfunden.

Die Metapher des Museums ruft nicht nur Assoziationen des Todes (Museum als Grab, Totenkammer usw.) ${ }^{14}$ in der Kollektivsymbolik hervor, sondern veranschaulicht ebenfalls die identitätsstörende Erfahrung der Selbstentfremdung, denn Identität wird stets auf der Grundlage von Erinnerungen gebildet: ,weil unsere Kindheit ein Museum ohne Namen ist, fehlen mir die Worte dafür; weil das Haus keine Adresse hat, weiß ich nicht, welchen Weg ich einschlagen soll, und komme in keiner Kindheit mehr an". ${ }^{15}$ Avanciert für Hensel das Schreiben zum einzig möglichen Modus des Rückbesinnens, das dem in den Anpassungsforderungen implizierten und zugleich internalisierten Gebot des Vergessen ${ }^{16}$ standhält, so wird der Text selbst zum Museum, in dem einerseits das Vergangene konser-

${ }^{12}$ Vgl. Caspari, Martina: Die schwierige Konstitution von Identität zwischen den Welten: Jana Hensels „Zonenkinder“. In: The German Quarterly 81/2008, S. 203-219.

${ }^{13}$ Mendívil, Julio: Ein musikalisches Stück Heimat: Ethnologische Beobachtungen zum deutschen Schlager. Bielefeld 2015, S. 243.

${ }^{14}$ Vgl. auch Hensel ebd., S. 20: „Ein bisschen sehen sie [alte Bekannte, Anm. d. Verf.] unter dem Glas wie Tote aus, und auf einmal bin ich nicht mehr ganz sicher, ob sie jemals unsere Freunde und wir mit ihnen am Leben gewesen sind“".

${ }^{15}$ Ebd., S. 25.

${ }^{16}$ Ebd., S. 14: ,[...] denn lange wollten wir sie [die „Märchenzeit“ der Vergangenheit, Anm. d. Verf.] vergessen, wünschten uns nichts sehnlicher, als dass sie so schnell wie möglich verschwinden würde. Es 
viert, andererseits dem Subjekt als Besucher/Betrachter die unüberbrückbare Distanz gegenüber den Exponaten stets bewusst gemacht wird.

Peter Weichharts sozialgeografischer Theorie zufolge entsteht die raumbezogene Identität, wenn sich ein Ego mit einem „abgegrenzten Raumgegenstand identifiziert, ihn damit zum Element der eigenen Ich-Identität macht und Gefühle der Zuneigung und Loyalität entwickelt" ${ }^{\prime \prime}{ }^{17}$ Aus dieser Sicht kann Hensels Text als Bericht der qualvollen Auseinandersetzung mit den Hürden einer sich primär über ihre Raumbezogenheit definierenden persönlichen Identitätsbildung angesehen werden, deren Übertragung auf die kollektive Ebene ohne jegliche Problematisierung erfolgt. ${ }^{18}$

Als prototypisch für Hensels Erzählstrategie mag die unterschiedliche Bilder gegeneinander ausspielende Darstellung des Tagebaus gelten. Nach der Wende stellt der Tagebau als physisch begehbarer, im Süden von Leipzig liegender Ort eine desolate Landschaft dar, wo „Kräne und Förderbänder, die teilweise verrostet und untätig, als seien sie vergessen worden, herumstanden, [...] während in einiger Entfernung noch immer die letzten Arbeiter geschäftig nach Braunkohle baggerten" ${ }^{19}$ Die Kindheitserinnerungen der dort geborenen Hensel lassen

war, als durfte sie nie existiert haben und als schmerzte es nicht, sich von Vertrautem zu trennen“.

${ }^{17}$ Weichhart, Peter: Grenzen, Territorien und Identitäten. In: Heintel, Martin/Robert Musil/Norbert Weixlbaumer (Hgg.): Grenzen. Theoretische, konzeptionelle und praxisbezogene Fragestellungen zu Grenzen und deren Überschreitungen. Wiesbaden 2018, S. 43-63, hier S. 60.

${ }^{18}$ Der von Hensel stillschweigend vorgenommene Transfer vom Ich zum Wir gehört zu den am meisten kritisierten Aspekten ihres Textes. Zur Rezeption von Zonenkinder vgl. Beerich-Shahbazi, Jennifer: The Zonenkinder Debate: An Analysis of Media Reactionto Two Popular Memoirs Written by East Germany's Youngest Generation of Authors. In: Backmann, Donald/Aida Sakalauskaite (Hgg.): Ossi Wessi. Newcastle 2008, S. 57-74.

${ }^{19}$ Hensel, ebd., S. 31. 
die Gegend hingegen als räumlich-soziales Milieu in ihrer früheren Dynamik wieder aufleben:

Ich kannte den Anblick und obendrein viele Leute, die jeden Morgen auf der einzigen Straße, die am Rand der Abraumtäler entlang ins Chemiewerk geführt hatte, zur Arbeit gefahren waren. Auf der anderen Seite der Grube lag mein Gymnasium. Es war erstaunlich, dass sie es überhaupt stehen gelassen hatten. Gleich hinter der Sporthalle begann der Tagebau. ${ }^{20}$

Die Nähe zum Tagebau und ihre Auswirkungen auf den Alltag (Knirschen des Sandes zwischen den Zähnen, Bestäubung der Fahrradrahmen usw.) war für die auf dem Schulhof spielenden Kinder oder die Internatsschüler Teil einer sich außerhalb der Ideologie abspielenden Normalität, die Hensel mit eindeutig provokatorischer Absicht ,recht ostentativ in Szene zu setzen“21 wagt. Auch auf der Ebene der sozialen Symbolisierung lassen sich dem Tagebau als imaginiertem Ort zwei entgegengesetzte Vorstellungen zuweisen, an denen der Konflikt zwischen dem hegemonialen Diskurs der Westdeutschen, in dem ostdeutsche Lebensformen und Sichtweisen einer Delegitimierung unterzogen wurden, und der Selbstwahrnehmung der Ostdeutschen, die auf diese Entwertung mit der hartnäckigen Verteidigung der eigenen Erfahrungen und Biografien reagierten, deutlich gemacht wird. Auf einem Spaziergang mit westdeutschen Gästen empfindet Hensel höchste Irritation, als dem Tagebau (ab)wertende Etiketten und vereinfachende politisierende Klischees angeheftet werden, durch die ihre Biografie ,,auf eine Hand voll Anekdoten“"22 reduziert wird. ,Raubbau“, „Boomtown“, ,,verantwortungslose Wohnungsbaupolitik" usw. sind Begriffe, die

${ }^{20}$ Ebd.

${ }^{21}$ Ledanff, Susanne: Neue Formen der „Ostalgie“ - Abschied von der „Ostalgie"? Erinnerungen an Kindheit und Jugend in der DDR und an die Geschichtsjahre 1989/90. In: A Journal of Germanic Studies 43/2007, S. 176-193, hier S. 179.

${ }^{22}$ Hensel, ebd. 
- wie die eigenen entfremdenden Erfahrungen der räumlichen Transformationen nach der Wende - sich mit der leiblich-affektiv erlebten Topographie ihrer Kindheit wenig decken:

Unsere Kindheit hatte weder in einer Boomtown noch in einer Mondlandschaft stattgefunden, sie kannte weder einen Raubbau noch eine verantwortungslose Wohnungsbaupolitik. Wir waren auf Wäscheplätzen, in Hinterhöfen, unter Kastanienbäumen und Pergolas oder auf Rollschuhbahnen zu Hause gewesen, und zwischen den weiß, gelb oder rosa sanierten Wohnhäusern, den gläsernen Bürokomplexen, den stählernen Monumenten des Industriezeitalters und den eintönigen Ladenstraßen der neunziger Jahre hatten wir nichts erlebt. ${ }^{23}$

Im Spannungsverhältnis zwischen der idyllischen Welt der verlorenen Kindheit und der dynamischen Zeit des Wandels entsteht das Bild der Ex-DDR als $Z$ wischenraum ${ }^{24}$, in dem die Identitätsbildung der Zonenkinder schwierig und in einer ersten langen Phase nur als Imitation des Westens stattfinden konnte. Trotzdem wird am Ende des Textes angedeutet, dass die Entwicklung in Richtung Identitätsbildung im wiedervereinigten Deutschland erfolgreich sein kann: „Es gibt hier heute nur noch sehr wenig, was so aussieht, wie es einst ausgesehen hat. Es gibt nichts, was so ist, wie es sein soll. Doch langsam fühlen wir uns darin wie zu Hause“. ${ }^{25}$

\section{Scotch(Klebeband) von Ioana Bradea: die verlorene Heimat als Ruinenlandschaft}

1975 in Bistrița geboren, zählt Ioana Bradea zu einer Generation junger Schriftsteller, die in den 2000er-Jahren debütiert und auf die turbulente historische Erfahrung der Wendezeit mit neuen

${ }^{23}$ Ebd., S. $31 \mathrm{f}$.

${ }^{24}$ Vgl. hierzu Caspari. Casparis aufschlussreiche Analyse zieht in Anlehnung an Homi K. Bhabha die Begriffe „Zwischenraum“ und ,mimicry“ für Hensels Zonenkinder heran.

${ }^{25}$ Vgl. auch Hensel, ebd., S. 155. 
Formen des Erzählens geantwortet haben. Ihr erster, 2004 erschienener Roman Băgău stellt einen der sowohl beim Publikum als auch bei der Kritik erfolgreichsten wie auch umstrittensten Romane der rumänischen zeitgenössischen Literatur dar. ${ }^{26}$ Auf weniger Resonanz ist ihr zweiter Roman Scotch gestoßen, wohl auch wegen des stark experimentellen Charakters des Textes, der weder herkömmliche Erzählinstanzen, noch individualisierte, gut entwickelte Figuren aufweist. Dadurch gelingt es Bradea allerdings, meiner Meinung nach, einen innovativen Blick auf die postkommunistischen Befindlichkeiten einer orientierungslosen Gesellschaft zu werfen, deren politische, wirtschaftliche und kulturelle Lebensformen eine riesige strukturelle Umwälzung erfahren haben.

Im Mittelpunkt des Romans steht „,das Bild des desolaten postindustriellen Raums einer Provinzstadt" ${ }^{\prime 27}$ wie man auf dem Rückumschlag des Buches lesen kann. In einer lyrischen Sprache, die eine Vielzahl poetischer Stilmittel mit präzisen, nüchtern-protokollierenden Aufzeichnungen verknüpft, wird die postkommunistische Ruinenlandschaft eines einst blühenden Industriegebiets geschildert. Das Kartographieren entsteht durch sich immer weiter ausdehnende Wahrnehmungskreise, die von den zwei Kilometer von der kleinen Stadt entfernten Gewerbeansiedlungen (Betrieb für mechanische Bearbeitungen, Kombinat für Holzbearbeitung, Werkzeugfabrik, Gießerei usw.) ausgehen, Schritt für Schritt wichtige Elemente der Stadt (die Zefirul-Straße mit dem Ledigenheim, ein äußerer Stadtteil mit der Narciselor-Straße, wo sich der Kneipenkomplex „La Betoane" befindet, Bahnhof, Friedhof, Laden für Elektrowaren

${ }^{26}$ Das mit dem Debüt-Literaturpreis des Rumänischen Schriftstellerverbandes ausgezeichnete Werk hat sich als Grundpfeiler der rumänischen erotischen Prosa wie auch des autofiktionalen Schreibens, das eine dominante Rolle in der aktuellen literarischen Landschaft einnimmt, etabliert.

${ }^{27}$ Alle Übersetzungen aus dem Rumänischen stammen von der Verfasserin. 
usw.) einbeziehen, um zurück zum Ausgangspunkt zu finden. Als zentrales Symbol der Industriezone fungiert der 200 Meter hohe Turm des Heizkraftwerks, dessen wiederholtes Erscheinen die Funktion eines allgegenwärtigen Zeugens übernimmt.

Das dominante Erzählprinzip scheint aus dem von Alain Robbe-Grillet entwickelten poetologischen Programm des nouveau roman entlehnt zu sein: Es herrschen exakte und detaillierte Beschreibungen von (oft insignifikanten) Gegenständen vor, die in der Art fotografischer Momentaufnahmen eine objektiv wirkende Bestandsaufnahme des Realen sichern. Als Darstellungsform wird häufig die Camera-Eye-Technik eingesetzt, bei der ein neutraler, unpersönlicher Blick kaleidoskopisch Realitätsbruchstücke registriert:

Pe jos, în dreptul tabloului de comandă, zac împrăștiate și roase cîteva chiștoace de țigări.

O cheie franceză uriașă, pe jumătate prinsă sub pata de vopsea albă de ulei împrăștiată pe ciment.

Cîteva capete de sîrmă galbenă și albastră.

Un creion chimic cu piele roșie și vîrf tocit.

Carcasa goală a unei unități de calculator - transformată în măsuță pentru masa de prînz. ${ }^{28}$

Allerdings dient dieses filmische Erzählverfahren im nouveauroman zur Ent-Anthropomorphisierung und Verdinglichung der Welt, die mit der Darstellung des Menschen als „Figur ohne

${ }^{28}$ Bradea, Ioana: Scotch. Iaşi 2010, S. 172: „Unten vor der Schalttafel liegen verstreut einige weggeworfene, alte Zigarettenstummel. Ein riesiger Schraubenschlüssel, im weißen Ölfarbenfleck auf dem Zementboden halb angeklebt. Ein paar gelbe und blaue Drahtenden. Ein abgestumpfter Kopierstift, mit rotem Leder bedeckt. Das leere Gehäuse eines Rechners, als Tischlein für das Mittagessen dienend.“ 
Tiefe“"29 einhergeht. ${ }^{30}$ In diesem entscheidenden Punkt weicht Bradea vom ästhetischen Modell des nouveau roman ab.

Die zweite zentrale Beschreibungstechnik basiert im Scotch auf dem vorherrschenden Stilmittel der Personifikation. Allem Leblosen, den Gebäuden der Industriezone, Maschinen, Werkzeugen bis zu Elementen der heruntergekommenen Landschaft und der Stadt, wird auf diese Weise ein dem Verfall bestimmter, aus Fleisch, Knochen, Eingeweiden usw. bestehender Organismus sowie eine Subjektivität zugeschrieben, die den allgemeinen Zerfallprozess des alten ökonomischen und sozialen Systems, die Verwahrlosung und allmähliche Zersetzung der Welt zu reflektieren vermögen. Statt des ,menschlichen Nichts des Gegenstandes“ („,le rien humain de l'objet”), ${ }^{31}$ das der Blick bei Robbe-Grillet instituiert, wird bei Bradea gerade die vermenschlichte Dingwelt zum zentralen erlebenden und erleidenden Subjekt tiefgreifender sozialer Umbruchprozesse, was das Ausmaß des historischen Traumas umso schärfer in den Vordergrund stellt. Die ehemalige Brotfabrik ,,a fost violată și părăsită mai demult" ",32 die Tür hängt lose in einer einzigen Angel, ,ca o gură muribundă uitată întredeschisă“", ${ }^{33}$ die Industriehalle mit nackter, wunder Brust zeigt ihre blutigen Ziegelsteine ${ }^{34}$, um nur

${ }^{29}$ Vgl. Stanzel, Franz: Theorie des Erzählens. Göttingen ${ }^{8}$ 2008, S. 297. In der klassischen Studie Stanzels wird das narrative CameraEyeVerfahren analysiert und der Technik des inneren Monologs gegenübergestellt.

${ }^{30}$ Tatsächlich hat der Diskurs bei Robbe-Grillet die Funktion, ,,alle Subjekte, Freiheit, Bewegung und Leben auszulöschen und in physikalischunbelebte Phänomene zu verwandeln, kurz: mögliche Entwicklung und Geschichte zugunsten von Physik und Geometrie auszulöschen.“ Vgl. Rother, Michael: Das Problem des Realismus in den Romanen von Alain Robbe-Grillet. Heidelberg 1980, S. 316.

${ }^{31}$ Barthes, Roland: Essais critiques. Paris 1964, S. 359.

${ }^{32}$ Bradea, Ioana: Scotch. Iaşi 2010, S. 63: „wurde vergewaltigt und längst verlassen".

${ }^{33}$ Ebd., S. 67: ,wie ein sterbender, halboffen vergessener Mund“.

${ }^{34}$ Vgl. ebd., S. 32. 
ein paar Beispiele für die Metaphorik der Gewalt zu erwähnen, die erlittenes historisches Leid am verstümmelten Körper festzumachen sucht.

Gegenübergestellt wird eine Fülle von non-humanen Akteuren der Quasi-Abwesenheit der Figuren - sogar die am meisten individualisierten Gestalten, die Sekretärin und der Ingenieur, bleiben bestenfalls skizzenhaft. Demnach erscheinen die Menschen als gesichtslose Masse, als verlorene, ohnmächtige Opfer historischer Mächte, deren Schicksal durch Verlust, völlige Perspektivenlosigkeit und Resignation gekennzeichnet ist. Das existenzielle Ausgeliefertsein wird im Bild der Sträflinge, mit dem das Buch einsetzt, treffend erfasst: Da die Fabrik neben der Strafanstalt gebaut wurde, tauschen die im Hof zum Schneefegen eingesetzten Sträflinge und die Arbeiter, die ihren Arbeitstag beginnen, Begrüßungsfloskeln aus.

Nur an wenigen Stellen wird dieses Trauma ausdrücklich thematisiert. Bei der Beschreibung der einzigen, in der Mitte der ehemaligen Produktionshalle übrig gebliebenen Maschine - das mehrere Tonnen schwere Gerät konnte nämlich nicht weggerissen werden - wird angemerkt: „Tuburile flutură murdar și s-ar ciocni între ele dacă ar bate vântul. Și-ar zdrăngăni istoria de făină albă și comunistă. ${ }^{35}$ Die Metapher , ,weißes kommunistisches Mehl" benennt das Scheitern der großen Utopie, ohne dass über Ursachen und Entwicklungen, über Schuld und Verantwortung geurteilt wird. ${ }^{36}$ Wie Pusca in ihrer Untersuchung zu Fotografie und Industrieruinen im postkommunistischen Europa argumentiert, können verfallene Räume wie Industrieruinen oft in dystopische Raum- und Zeitvorstellungen überführt werden:

${ }^{35}$ Ebd., S. 113: „Die schmutzigen Schläuche zittern und bei Wind würden sie gegeneinander stoßen. Sie würden ihre Geschichte von weißem kommunistischem Mehl klappernd kundtun“.

${ }^{36}$ Vgl. Mironescu, Doris: Un secol al memoriei. Literatură și conştiințăa comunitară în epoca romantică. Iaşi 2016, S. 302, der hier allerdings eine antikommunistische Stellung seitens des impliziten Erzählers zu entdecken meint. 
If, as Susan Buck-Morss eloquently explains, spaces and buildings were used to visually inscribe the communist utopia into people's minds, the ruination of these same spaces can be directly connected to the dismantling of that utopia. Along with the buildings, it was not just an ideology - mass utopia or dream world as Buck-Morss calls it - that went crumbling, but also a particular collective and individual identity, a social order that sustained life both physically - through the industrial platforms and the cities surrounding them - as well as emotionally - through the communities and solidarity that emerged within them. ${ }^{37}$

Derselbe distanzierte, jedoch subtil ironische Umgang mit der Geschichte lässt sich bei der Gegenüberstellung von gegenwärtigen Trümmern und den ihrerzeit glorreich gefeierten Errungenschaften des Sozialismus feststellen. Im Roman wird in Kursivschrift ein 1972 in der lokalen Zeitung erschienener Artikel über die Errichtung des Kombinats für Holzbearbeitung eingebunden, das als „neuestes Werk des Aufbaus des Sozialismus“ einen Meilenstein auf dem Weg in die lichtvolle Zukunft darstellen soll: „Muncitori, tehnicieni, maiștri, ingineri - un nucleu de prospețime și optimism: sunt veseli, înflăcărați, încrezători în forțele și cunoștințele lor. Locul de muncă - Fabrica de Mobilă - pe toți îi unește. Cariere și destine îşi deschid aici aripi tinerești". 38

Die zeitliche Dimension entfaltet sich ausgehend von dieser fundamentalen Entgegensetzung zwischen Vergangenheit und Gegenwart, die entweder implizit im Bild des gegenwärtigen Zerfalls mitsignalisiert, oder explizit durch direkten Bezug auf den ehemaligen Zustand bewerkstelligt wird. Im letzteren Fall

${ }^{37}$ Pusca, Anca: Industrial and Human Ruins of Postcommunist Europe. In: Space and Culture 13/2010, S. 239-255, hier S. 241.

${ }^{38}$ Bradea, ebd., S. 84: „Arbeiter, Techiker, Meister, Ingenieure - ein Kern von Frische und Optimismus: Sie sind froh, zeigen eine große Begeisterung und viel Vertrauen in ihre eigenen Kräfte und Kenntnisse. Ihr Arbeitsplatz - die Möbelfabrik- verbindet sie alle. Der Karriere und dem Schicksal werden hier junge Flügel verliehen“. 
dienen personifizierende Verben häufig der Konstruktion zeitlicher Rückwendungen: „Manche von ihren Wänden erinnern sich noch daran" ${ }^{39}$ Der Einsatz des Präsens als vorherrschende Zeitform der Erzählung hat selbstverständlich mit der fotografischen Beschreibungsstrategie zu tun. Doch so wie der fotografische Akt - Roland Barthes zufolge - die Tötung des Subjekts ${ }^{40}$ voraussetzt, verweist dieses Präsens auf eine im Verfall begriffene Welt, deren Geschichte nur noch als traumatisch erfahren wird. Steht der (historische, soziale, biologische, materielle) Wandel im Mittelpunkt des Romans, so ist dieser Wandel stets als Ver- und Zerfall repräsentiert und in der symbolischen Form der dystopischen Ruineverfestigt.

Für Mironescu, der das Aufkommen einer neuen postkommunistischen Ruinenliteratur im Vergleich zu ihren romantischen Vorläufern analysiert, scheint in solchen (Nach-)WendeProsawerken wie das von Bradea ein postkanonisches

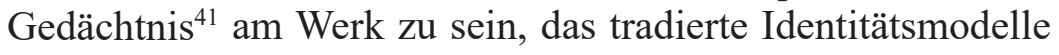
hinterfragt, ohne eindeutige und/oder einheitliche Antworten vorzuschlagen. ${ }^{42}$ Tatsächlich verweist die Titelmetapher Klebeband auf den Versuch einer zerbrochenen und zerrissenen Gesellschaft, durch ,Flicken' und punktuelles Aufbessern das Vergangene zu retten, in einer sinnentleerten Gegenwart voranzukommen und ihr Überleben zu sichern. Auf Zeichen möglicher künftiger Entwicklungen wird im Roman ab und zu einge-

${ }^{39}$ Ebd., S. 83: „Unii dintre pereții lor încă își amintesc“.

${ }^{40}$ Barthes, Roland: Die helle Kammer: Bemerkung zur Photographie. Übersetzt von Dietrich Leube. Berlin 1989, S. 24: „Der Tod ist das Eidosdieser Fotografie vor meinen Augen."

${ }^{41}$ Der Begriff ,postkanonisches Gedächtnis“ geht auf Jan Assmanns (2017: 18) Auffassung einer, ,postkanonischen Kultur“ zurück, ,deren Inhalte ihren verpflichtenden Charakter und ihre bindende Kraft aufgegeben haben." Vgl. Assmann, Jan: Das kulturelle Gedächtnis: Schrift, Erinnerung und politische Identität in frühen Hochkulturen. München 2017.

${ }^{42}$ Vgl. Mironescu, ebd., S. 296-304. 
gangen, doch sieht die Zukunft genauso unsicher und düster aus wie die von Profit gesteuerte Gegenwart. Neben der ehemaligen, nun verlassenen Schnapsfabrik wird eine neue Fabrik für Tiefkühl-Schnecken gebaut, doch nichts scheint auf Erfolg zu deuten: „Dar cine va culege cele cinci mii de tone de melci/ De unde anume" ${ }^{43}$ Die einzigen hellen Momente gehören einer idealisierten Vergangenheit an, in der die traditionelle, harmonische und naturverbundene Bauernwelt, die infolge Ceauşescus Urbanisierungsprojekt einer forcierten Modernisierung unterworfen wurde, noch existierte.

\section{Fazit}

Sowohl Hensel als auch Bradea schreiben über das Verschwinden der alten Lebensformen, über gewaltige Transformationsprozesse auf politischer, sozialer, kultureller und ökonomischer Ebene, über das Gefühl der Orientierungslosigkeit in einer Transitionsgesellschaft, in der alte und neue Wertvorstellungen und Verhaltensmuster miteinander kollidieren. Allerdings greifen die Autorinnen zu unterschiedlichen, ja geradezu entgegengesetzten Erzählstrategien. Während bei Hensel das kollektive Gedächtnis einer Schicksalsidentität von der klagenden WirStimme getragen wird, erfolgt die Darstellung historischer Prozesse bei Bradea nicht vorrangig über Figuren, sondern über die Lebensgeschichten von Gegenständen, wobei die neutrale Erzählinstanz im Kamera-Modus das Scheitern der Ideologien unbeteiligt und kommentarlos registriert.

\section{Literatur}

\section{Primärliteratur}

Bradea, Ioana: Scotch. Iaşi 2010.

Hensel, Jana: Zonenkinder. Reinbek bei Hamburg 2002.

${ }^{43}$ Bradea, ebd., S. 56: „Aber wer wird die fünftausend Tonnen Schnecken sammeln / und woher". 
Roxana-Andreea Ghiță

\section{Sekundärliteratur}

Assmann, Jan: Das kulturelle Gedächtnis: Schrift, Erinnerung und politische Identität in frühen Hochkulturen. München 2017.

Bahr, Katrin/Lorek, Melanie: „Ja, wohingehen sie denn?“: die „3. Generation Ostdeutscher" zwischen Suchen und Finden am Beispiel des 1.5 Generationskonzeptes. In: Lettrari, Adriana/Christina Nestler/Nadja Troi-Boeck (Hgg.): Die Generation der Wendekinder: Elaboration eines Forschungsfeldes. Wiesbaden 2015, S. 255-278.

Barthes, Roland: Essais critiques. Paris 1964, S. 359.

Barthes, Roland: Die helle Kammer: Bemerkung zur Photographie. Übersetzt von Dietrich Leube. Berlin 1989.

Beerich-Shahbazi, Jennifer: The Zonenkinder Debate: An Analysis of Media Reaction to Two Popular Memoirs Written by East Germany's Youngest Generation of Authors. In: Backmann, Donald/Aida Sakalauskaite (Hgg.): Ossi Wessi. Newcastle 2008, S. 57-74.

Caspari, Martina: Die schwierige Konstitution von Identität zwischen den Welten: Jana Hensels ,Zonenkinder“. In: The German Quarterly 81/2008, S. 203-219.

Ghiță, Roxana-Andreea: Literatur und Geschichte. Fallstudien zur narrativen Aufarbeitung der Vergangenheit im deutschen und europäischen Kontext. Craiova 2018.

Kraushaar, Tom: Die Zonenkinder und Wir - Die Geschichte eines Phänomens. Reinbek bei Hamburg 2004.

Ledanff, Susanne: Neue Formen der „Ostalgie“ - Abschied von der ,Ostalgie“"? Erinnerungen an Kindheit und Jugend in der DDR und an die Geschichtsjahre 1989/90. In: $A$ Journal of Germanic Studies 43/2007, S. 176-193.

Mendívil, Julio: Ein musikalisches Stück Heimat: Ethnologische Beobachtungen zum deutschen Schlager. Bielefeld 2015.

Mironescu, Doris: Un secol al memoriei. Literatură și conștiință comunitară în epoca romantică. Iaşi 2016. 
Müller, Klaus: Einleitung. In: Müller, Klaus (Hg.): Postsozialistische Krisen: Theoretische Ansätze und empirische Befunde. Wiesbaden 2013, S. 7-9.

Pusca, Anca: Industrial and Human Ruins of Postcommunist Europe. In: Space and Culture13/2010, S. 239-255.

Rother, Michael: Das Problem des Realismus in den Romanen von Alain Robbe-Grillet. Heidelberg 1980.

Steinig, Valeska: Abschied von der DDR - Autobiografisches Schreiben nach dem Ende der politischen Alternative. Frankfurt am Main 2007.

Stanzel, Franz: Theorie des Erzählens. Göttingen ${ }^{8} 2008$.

Sztompka, Piotr: The Ambivalence of Social Change in PostCommunist Societies. The Case of Poland. In: Soeffner, Hans-Georg (Hg.): Unsichere Zeiten. Herausforderungen gesellschaftlicher Transformationen. Wiesbaden 2010, S. 265-286.

Weichhart, Peter: Grenzen, Territorien und Identitäten. In: Heintel, Martin/Robert Musil/Norbert Weixlbaumer(Hgg.): Grenzen. Theoretische, konzeptionelle und praxisbezogene Fragestellungen zu Grenzen und deren Überschreitungen. Wiesbaden 2018, S. 43-63. 